\title{
Surface characterization of Nb samples electropolished with real superconducting rf accelerator cavities
}

\author{
Xin Zhao, ${ }^{1, *}$ Rong-Li Geng, ${ }^{1}$ P. V. Tyagi, ${ }^{2}$ Hitoshi Hayano, ${ }^{3}$ Shigeki Kato, ${ }^{2,3}$ \\ Michiru Nishiwaki, ${ }^{3}$ Takayuki Saeki, ${ }^{3}$ and Motoaki Sawabe ${ }^{3}$ \\ ${ }^{1}$ Thomas Jefferson National Accelerator Facility, Newport News, Virginia 23606, USA \\ ${ }^{2}$ Graduate University for Advanced Studies, School of Advanced Sciences (GUAS/AS), Ibaraki, Japan \\ ${ }^{3}$ High Energy Accelerator Research Organization (KEK), Ibaraki, Japan
}

(Received 30 November 2009; revised manuscript received 2 December 2010; published 30 December 2010)

\begin{abstract}
We report the results of surface characterizations of niobium $(\mathrm{Nb})$ samples electropolished together with a single cell superconducting radio-frequency accelerator cavity. These witness samples were located in three regions of the cavity, namely at the equator, the iris, and the beam pipe. Auger electron spectroscopy was utilized to probe the chemical composition of the topmost four atomic layers. Scanning electron microscopy with energy dispersive $\mathrm{x}$ ray for elemental analysis was used to observe the surface topography and chemical composition at the micrometer scale. A few atomic layers of sulfur (S) were found covering the samples nonuniformly. Niobium oxide granules with a sharp geometry were observed on every sample. Some $\mathrm{Nb}-\mathrm{O}$ granules appeared to also contain sulfur.
\end{abstract}

DOI: 10.1103/PhysRevSTAB.13.124702

PACS numbers: $81.65 . P s$

\section{INTRODUCTION}

In 1931, Jacquet discovered that electropolishing (EP) can efficiently reduce roughness of a metal surface by applying the metal as anode [1]. Since the first successful electropolishing of niobium cavities in the 1970s [2], many important developments of this technique have been made over the past decades. Saito's work demonstrated that electropolishing is a necessary surface processing method for high gradient superconducting radio-frequency cavities made of bulk niobium [3]. The most notable progress in the EP method is the implementation of the horizontal EP technique developed by KEK and Nomura Plating [3]. Horizontal EP machines are now installed in many laboratories as well as in the industry. In pursuit of a further understanding of the EP mechanism, modern studies addressed many detailed aspects of this useful process [4-8]. The conventional electrolyte for EP is a mixture of hydrofluoride and hydroxide sulfuric acids using a volume ratio of 1:10 [HF (49\%): $\mathrm{H}_{2} \mathrm{SO}_{4}(96 \%)$ ]. Large amounts of yellow sulfur crystalline powders had been found at the outlet of the used EP electrolyte. In the EP process, the entire niobium $(\mathrm{Nb})$ cavity is an anode for polishing, and the cathode is made of aluminum. It is known that on the cathode surface, $\mathrm{SO}_{4}{ }^{2-}$ could be reduced to $\mathrm{S}$ [6-8]. Noer and Niedermann et al. used SEM, AES, and field emission scanning microscopy to study electron field emission from pure sulfur, $\mathrm{MoS}_{2}$, and carbon particles on a clean $\mathrm{Nb}$ surface $[9,10]$. They found that artificially introduced pure sulfur particles had very weak field emission. But the sulfur element detected at strong field emission sites

\footnotetext{
*Correspondent author. xinzhao@jlab.org
}

is often associated with another element, normally Mo (maybe from lubricants of mechanics). They suggested that layered materials, such graphite, $\mathrm{MoS}_{2}$, and $\mathrm{NbS}_{2}$ might favor field emission. For future work, they proposed that a detailed study of $\mathrm{Nb}-\mathrm{S}$ interaction, especially when impurities like Mo particles are presented, should be useful. We are interested to investigate the surface state of a real $\mathrm{Nb}$ cavity after EP via AES and SEM. The motivation of this study is to search for possible impurities left by the electropolishing process, which might enhance field emission and degrade cavities' field gradient limit. Specifically, this study was initiated to search for any sulfur residues on the $\mathrm{Nb}$ cavity surface immediately after EP. In this research three small witness samples have been processed in situ with a single cell superconducting radio-frequency (SRF) cavity (Fig. 1). The samples are round disks of $8 \mathrm{~mm}$ in

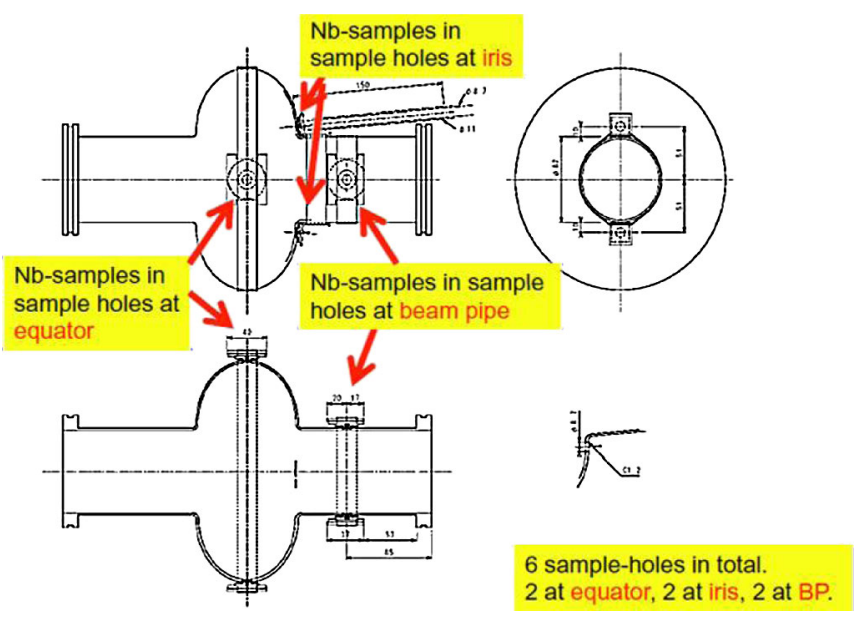

FIG. 1. Three witness samples were located in a single cell SRF cavity and electropolished in situ. 

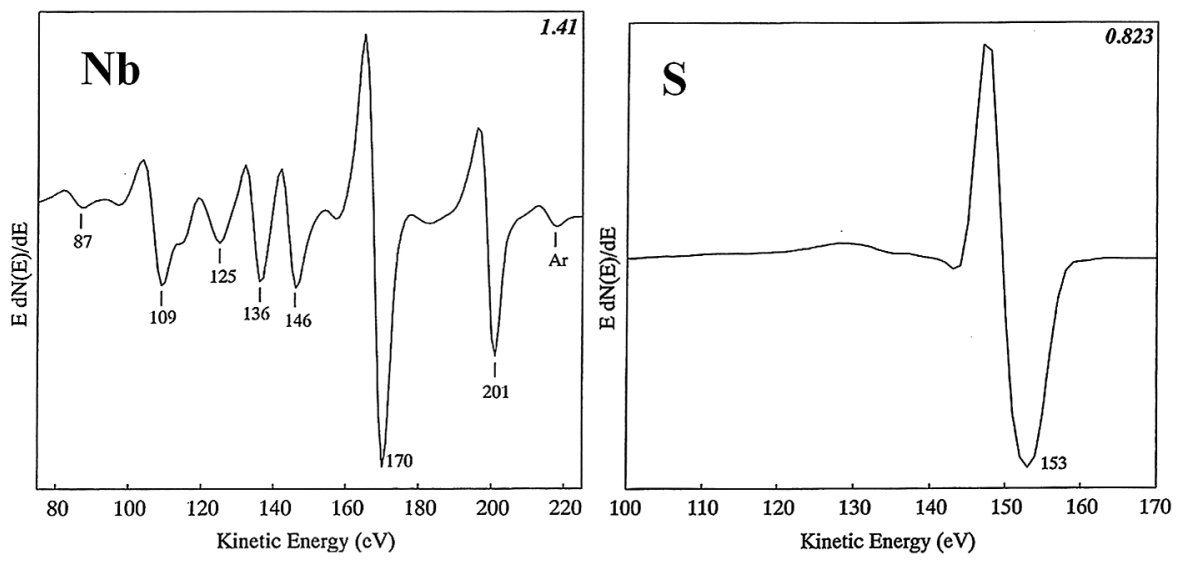

FIG. 2. Fingerprint Auger spectrum of niobium and sulfur [8].

diameter. They experienced the same EP (50 $\mu \mathrm{m}$ removal $)$ as the real cavity. They were rinsed by ultrapure water but without ultrasonic rinsing. The samples were located at iris, equator, and beam pipe, respectively. The entire EPrelated experiment was conducted at High Energy Accelerator Research Organization (KEK) in Japan.

\section{EXPERIMENTAL METHOD}

Auger electron spectroscopy (AES) was applied to study the chemical composition of the samples' topmost surface. AES (PHI 660 system made by Physical Electrons ${ }^{\mathrm{TM}}$ ) is sensitive to the first four atomic layers $(\sim 1.2 \mathrm{~nm})$. Its best sensitivity on atomic concentration is $0.2 \%$. This instrument has an absorption current imaging system (ABS), which roughly shows surface morphology and elemental mass. The ABS imaging system was used to identify the primary electron beam spot and the $\mathrm{Ar}^{+}$depth profiling ion beam spot. Figure 2 shows the AES "fingerprint" spectra of niobium (Nb) and sulfur (S) [11]. Their peak profile and peak energies were both used to search for sulfur in the empirical spectrum. One should note that the sulfur peak $(153 \mathrm{eV})$ is in the middle of two $\mathrm{Nb}$ peaks (146 and $170 \mathrm{eV}$ ). Since the $\mathrm{S}$ and $\mathrm{Nb}$ peaks are very close, peak assignment may be quite challenging. For all AES experiments in this study, if not said otherwise, the primary e-beam current is $100 \mathrm{nA}$ and acceleration voltage is $2 \mathrm{kV}$; the beam spot has a square shape $(250 \times 250 \mu \mathrm{m})$. The electron multiplier's (channeltron) voltage was automatically set to $1850 \mathrm{eV}$. The sweep rate was $1 \mathrm{eV} / \mathrm{s}$ and collecting dwell time was $50 \mathrm{msec}$. Each spectrum is an average of 20 sweeps.

To calibrate the AES instrument, molybdenum disulfur $\left(\mathrm{MoS}_{2}\right)$ powders were intentionally laid on a bufferchemical polished (BCP) niobium sample, and the sample was surveyed between 80-220 eV. In Fig. 3, the theoretical sulfur AES peak position is labeled by an orange solid line; the theoretical $\mathrm{Nb}$ AES peak positions are labeled by gray dashed lines. In Fig. 3, Nb spectrum (the top curve, in red) of the cleaned BCP surface is identical to the referenced
$\mathrm{Nb}$ fingerprint spectrum (Fig. 2). The spectrum (the blue bottom curve) of $\mathrm{MoS}_{2} / \mathrm{Nb}$ indicates a strong sulfur peak in the middle of the $\mathrm{Nb}$ peaks. This testing demonstrated that the AES system could discern the sulfur element.

A high resolution scanning electron microscope, Hitachi-4700, was used to observe surface morphology. It has an energy dispersive x-ray (EDX) detector made by $\mathrm{EDAX}^{\mathrm{TM}}$. Unlike AES, which uses emitted Auger electrons to identify elements, the EDX system utilizes characteristic $\mathrm{x}$-ray photons excited by the primary electron beam to probe chemical elements. It can explore $2 \mu \mathrm{m}$ in

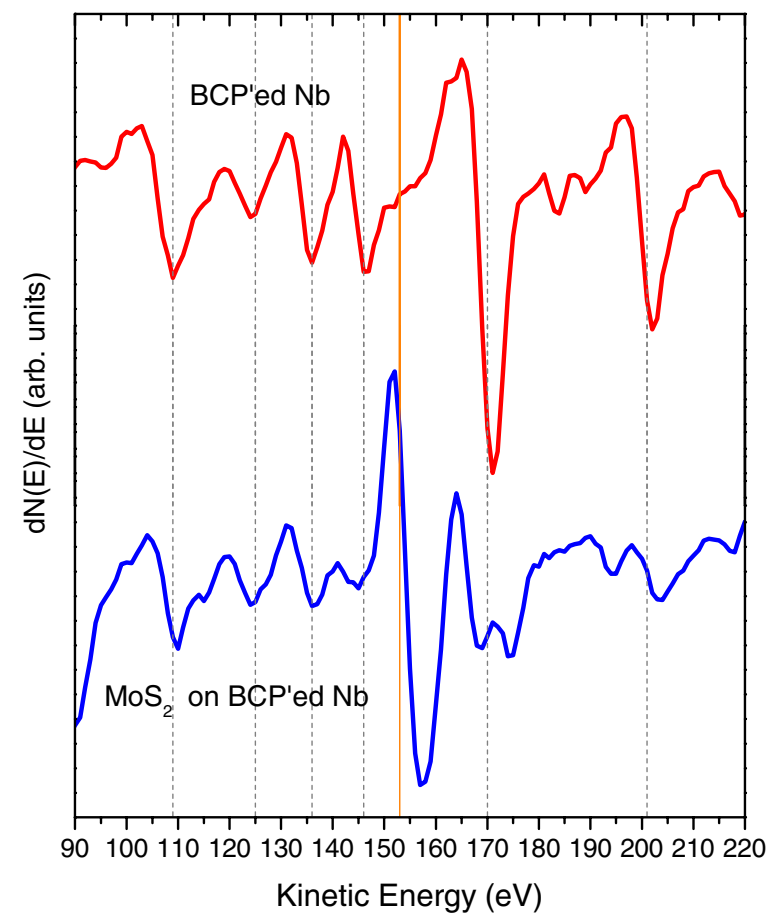

FIG. 3. AES spectrum (scaled) of a BCP niobium (red curve) and a $\mathrm{MoS}_{2}$-covered niobium surface (blue curve). The sulfur and niobium fingerprint peak positions are labeled by a solid orange line and gray dashed lines, respectively, for reading convenience. 
TABLE I. Characteristic x-ray photon energy and Auger electron kinetic energy of some elements.

\begin{tabular}{|c|c|c|c|c|c|c|}
\hline & $\mathrm{Nb}$ & $\mathrm{O}$ & $\mathrm{S}$ & $\mathrm{Fe}$ & $\mathrm{N}$ & $\mathrm{C}$ \\
\hline Characteristic x-ray photo energy (eV) & $\begin{array}{c}2164 \text { (La1) } \\
2254 \text { (La2) } \\
172(\mathrm{Mz} 1)\end{array}$ & $515(\mathrm{Ka} 1)$ & $\begin{array}{c}2308(\mathrm{Ka} 1) \\
149(\mathrm{LI})\end{array}$ & $\begin{array}{l}6402(\mathrm{Ka} 1) \\
705(\mathrm{La} 1)\end{array}$ & $384(\mathrm{Ka} 1)$ & $266(\mathrm{Ka} 1)$ \\
\hline Auger electron kinetic energy $(\mathrm{eV})$ & $\begin{array}{c}87 \\
109 \\
125 \\
136 \\
146 \\
170 \\
201\end{array}$ & 510 & 153 & $\begin{array}{l}589 \\
645 \\
700\end{array}$ & 375 & 272 \\
\hline
\end{tabular}

depth and has an atomic sensitivity $\sim 2 \%$. In contrast to AES (probing four atomic layers), EDX is a more "bulklike" elemental analysis tool. For EDX experiment in this study, the primary electron beam has acceleration voltage $15 \mathrm{kV}$, and the spectrum collection time was 200 seconds. For spectrum analysis convenience, some elements' characteristic x-ray photo energy and Auger electron energy is listed in Table I. The Nb La1 $(2164 \mathrm{eV})$ and La2 peaks $(2254 \mathrm{eV})$ are close together; their intensity ratio is 100:42. Because of their inherent peak widths and intensity ratio (100:41), two peaks seem like a single broad peak with a "shoulder" on the high energy side. One must carefully distinguish this feature from a sulfur peak at $2308 \mathrm{eV}$.

\section{RESULTS AND ANALYSIS}

\section{A. Results of sample at the equator zone (sample 2)}

Sample 2 was located at the equator. Five spots were surveyed by AES. The spots were 1 or $2 \mathrm{~mm}$ away from each other. No sulfur was found in any of the surveyed spots. As a representative, the spectrum collected at one

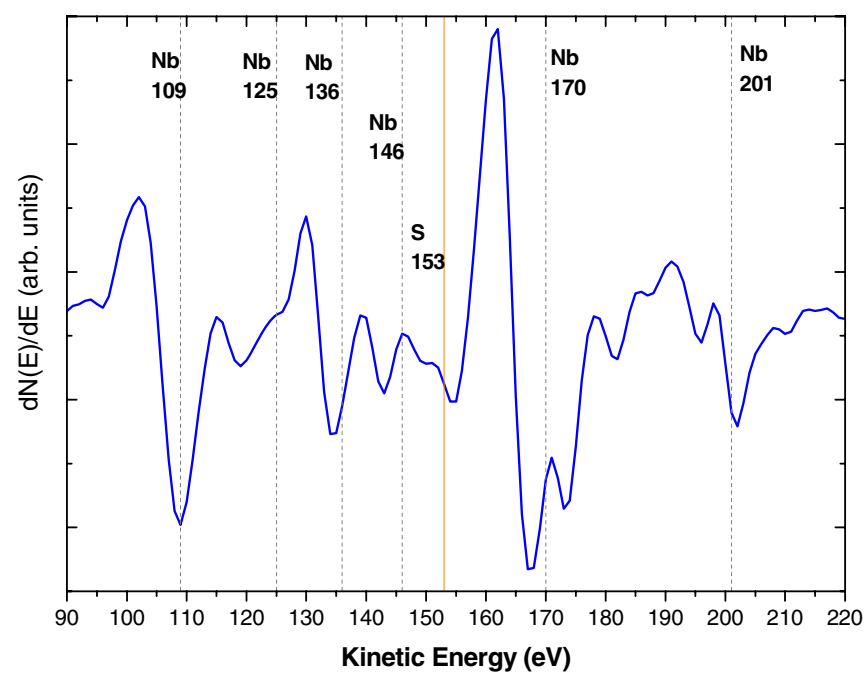

FIG. 4. AES spectrum of sample 2 (spot 5). No sulfur peak was found at $153 \mathrm{eV}$. The spectra surveyed at other spots indicate no sulfur either. spot (\#5) is shown in Fig. 4. At $153 \mathrm{eV}$ (sulfur position), no peak was observed.

Under SEM, granules (Fig. 5) were observed sparsely sitting on the largely smooth surface. The granules' morphology looks like sintered crystallites. It is noticed that the granules (e.g. Fig. 5) reside on a very smooth flat surface. It is not yet known if the granules drifted to the surface from the electrolyte or were formed in situ. Figure 6 is an EDX spectrum collected from a spot on a granule. The fingerprint positions of niobium, oxygen, sulfur, and carbon are labeled by short-dashed gray lines for peak identification convenience. Only $\mathrm{Nb}$ and $\mathrm{O}$ were found on the granule. Because the sampling depth of EDX is $1 \mu \mathrm{m}$, it is speculated that the granule is entirely niobium oxide, but not a naturally oxidized bulk niobium grain. For a niobium surface after natural atmospheric oxidization, the niobium oxide is only a few nanometers thick, which could only contribute a much weaker peak in an EDX spectrum.

\section{B. Results of sample at the iris zone (sample 4)}

Sample 4 was located at the iris area. Similar to sample 2, sparsely distributed $\mathrm{Nb}-\mathrm{O}$ granules were also observed on this sample. The granules' geometrical features and chemical concentration under SEM/EDX are similar to that found on sample 2 (shown as Fig. 5).

Five spots were surveyed by AES. They were separated by a distance $>2 \mathrm{~mm}$. Four spots had strong sulfur signals and one spot had none. For the four spots with sulfur, the atomic ratio of $\mathrm{Nb} / \mathrm{S}$ is $3 / 1,6.14 / 1,6.35 / 1$, and 2.98/1, respectively. Figure 7 is the spectrum observed at spot 1. It has a strong sulfur peak (label in red). The atomic concentration ratio of $\mathrm{Nb}$ to $\mathrm{S}$ is close to 3:1. Survey results from the five spots suggested that the sulfur coverage is not uniform however.

An argon ion beam was used to slightly sputter the surface. Figure 8 shows the AES spectra of the spot before and after sputtering. The beam current was $10 \mathrm{nA}$, beam spot area $\sim 27 \mathrm{~mm}^{2}$, and ion beam acceleration voltage $2 \mathrm{kV}$. The sputtering time was 10 minutes. The sputtering rate (or depth) was not empirically calibrated. 


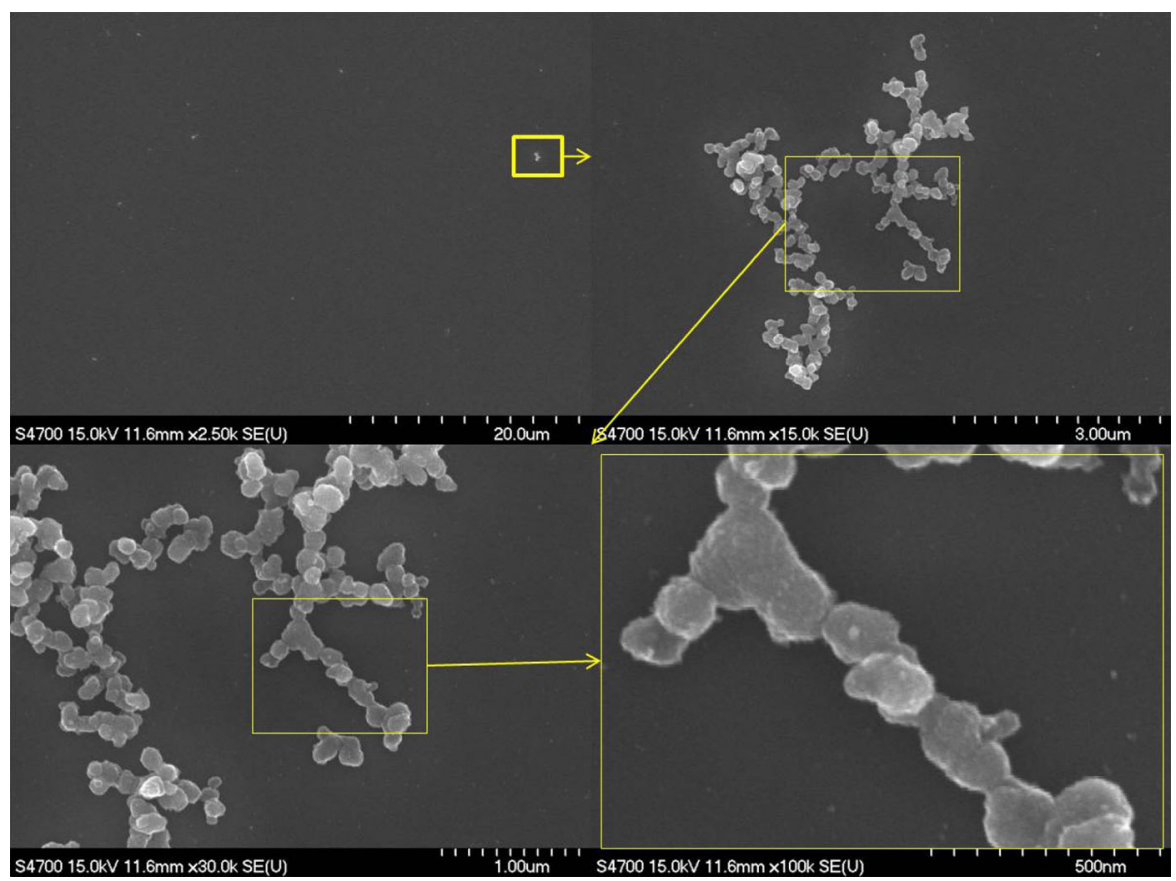

FIG. 5. Sparsely distributed granules were observed on the sample surfaces. They reside on a clear flat surface and their density is low.

Nevertheless, it was theoretically evaluated that the profiling depth was smaller than ten atomic layers. Before the sputtering (red curve in Fig. 8), the sulfur peak has an obvious profile $(\mathrm{Nb} / \mathrm{S} \sim 3: 1)$. After the sputtering (blue curve), the sulfur peak intensity becomes much smaller $(\mathrm{Nb} / \mathrm{S} \sim 10: 1)$. This suggests the sulfur formed an atomically thin layer on the outmost surface of the sample after electropolishing. It seems that the sputtering process

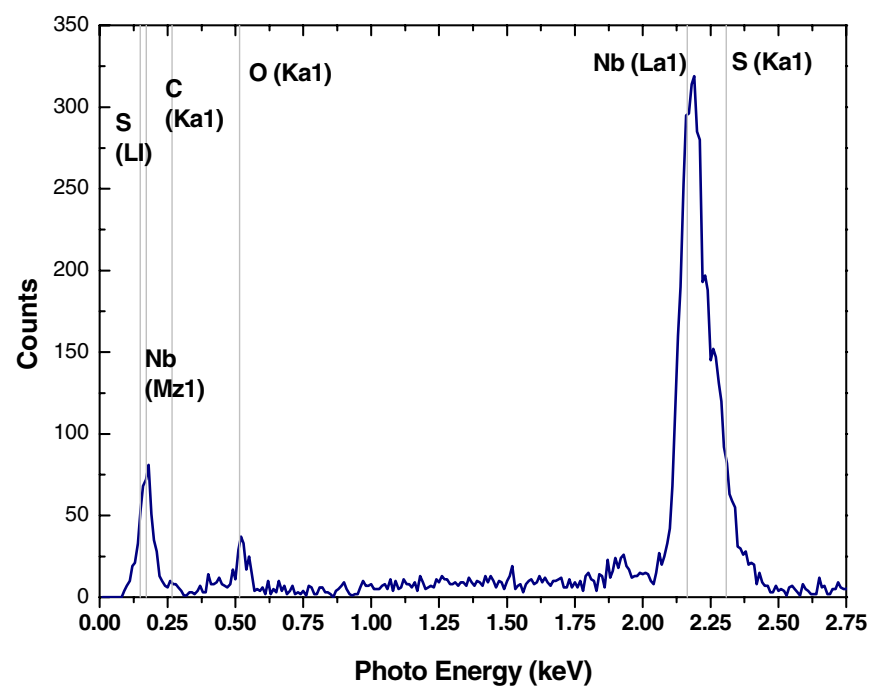

FIG. 6. EDX spectrum surveyed at a spot of the granule (as shown in Fig. 5, survey area $300 \times 200 \mathrm{~nm}^{2}$ ). Only oxygen and niobium elements are observed. The fingerprint positions of $\mathrm{Nb}$, $\mathrm{O}, \mathrm{S}$, and $\mathrm{C}$ are all labeled by gray lines for reading convenience. cleaned a surface dielectric layer (thus reduced the charging effect, Fig. 9).

\section{Results of sample at the beam pipe (sample 6)}

Sample 6 was located at the beam pipe. Eleven spots were surveyed via AES. On three of the 11 spots, sulfur was found (spectra similar to Fig. 7, not shown here). A variety of microgranules were investigated by SEM/EDX, and some of the granules morphology is similar to that observed



FIG. 7. AES spectrum observed on spot 1. The theoretical sulfur peak position is labeled in orange. The $\mathrm{Nb}: \mathrm{S}$ atomic ratio is 3:1 after RBD Instruments Augerscan ${ }^{\mathrm{TM}}$ quantification evaluation. 


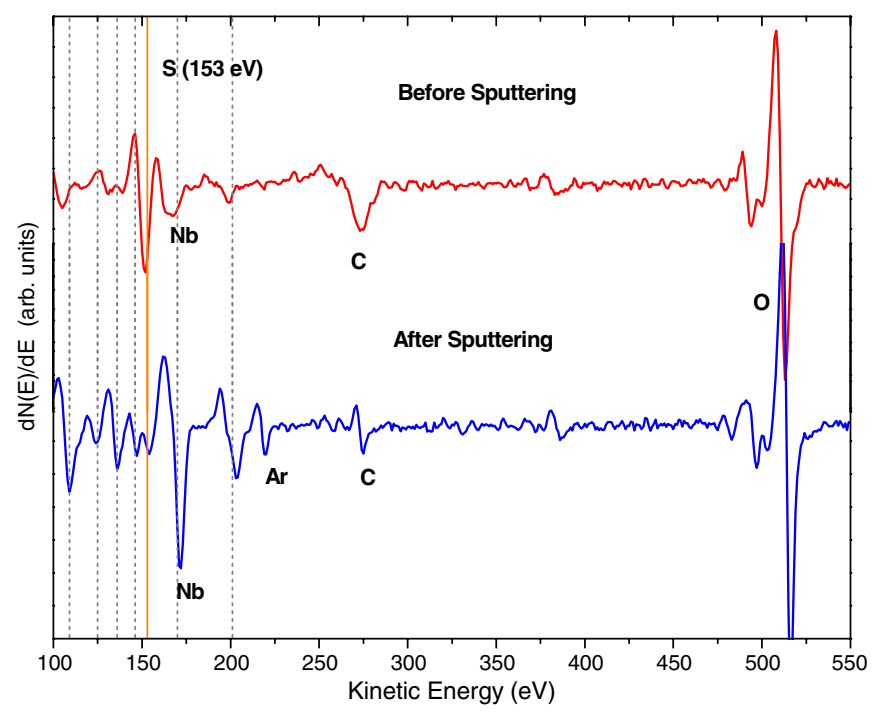

FIG. 8. AES spectra (scaled) of a spot before and after a slight sputtering. Fewer than ten atomic layers were removed by the $\mathrm{Ar}^{+}$beam. The sulfur peak was greatly reduced after sputtering, which indicated sulfur coverage is ultrathin.

on sample 2 (shown as Fig. 5). However, on one granule, EDX detected sulfur. Figure 10 shows the granule's SEM image. It lays on a smooth surface and was separated from other particles. The first EDX detection window (spot 1) is labeled by a yellow box, which is located at the upper-left corner in Fig. 10. The first EDX spectrum is presented by a blue curve in the top of Fig. 11. The gray dashed lines in Fig. 11 indicate fingerprint positions of various elements (e.g. sulfur at $2.3 \mathrm{keV}$ ). The blue curve shows only $\mathrm{Nb}$ and $\mathrm{O}$, but no sulfur. Nevertheless, the $\mathrm{O} / \mathrm{Nb}$ ratio is much higher than a naturally oxidized $\mathrm{Nb}$ surface.

The second EDX detection window (spot 2) is also labeled by a yellow box, which is located at the bottomright corner in Fig. 10. The EDX spectrum on spot 2 is

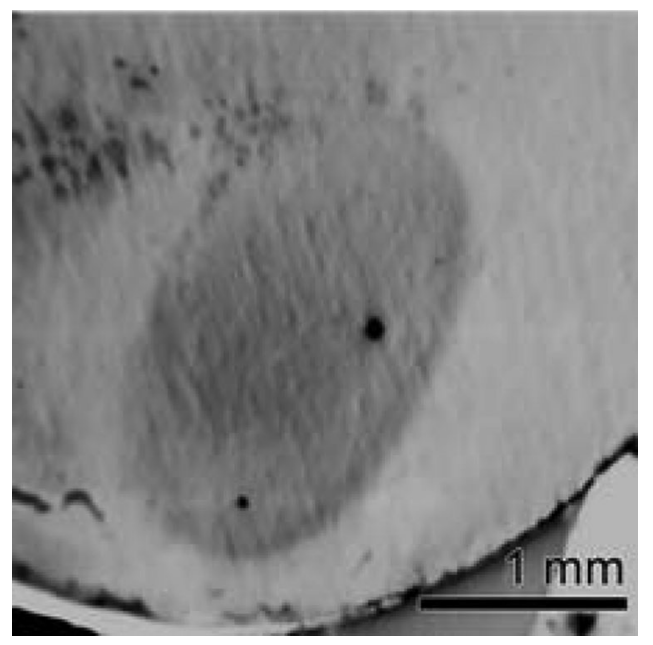

FIG. 9. ABS image of the sputtered spot (the elliptical-shaped grey zone). It seems the $\mathrm{Ar}^{+}$beam cleaned the surface layer (less charging effect after sputtering).

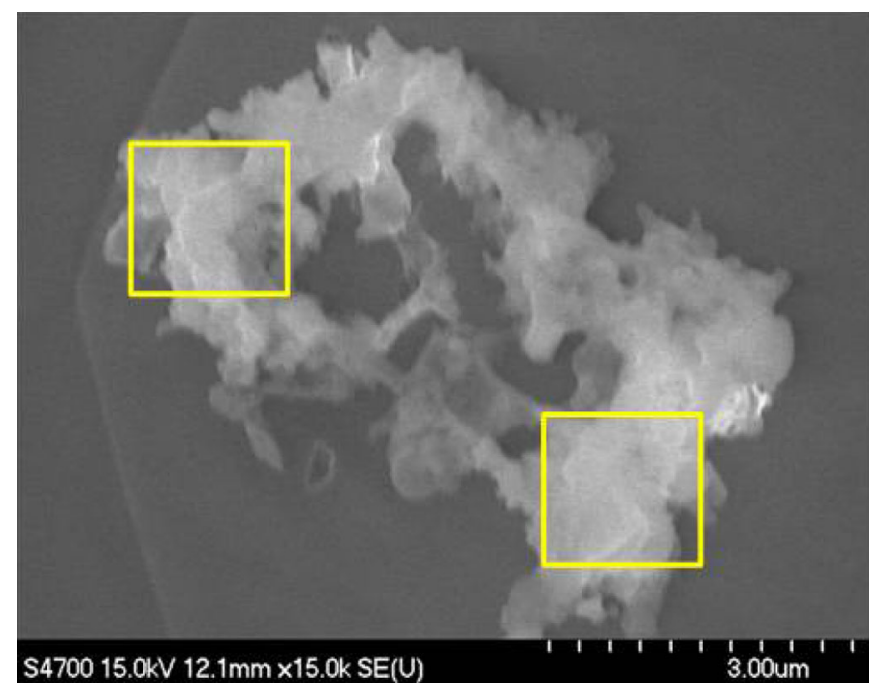

FIG. 10. A sulfur-embedded granule observed on sample 6 . Sulfur is enriched on one spot (spot 2, bottom-right yellow window) but not at another spot (spot 1, upper-left window). This granule is setting on a very smooth and flat plane.



FIG. 11. EDX spectra (scaled) from two spots on a granule (Fig. 10) and from the off-granule flat plane (50 um away from the granule). Sulfur is enriched on spot 2 (red curve); the oxygen to niobium ratio is much higher on the granule than that of a normal EP'ed surface (black curve). 

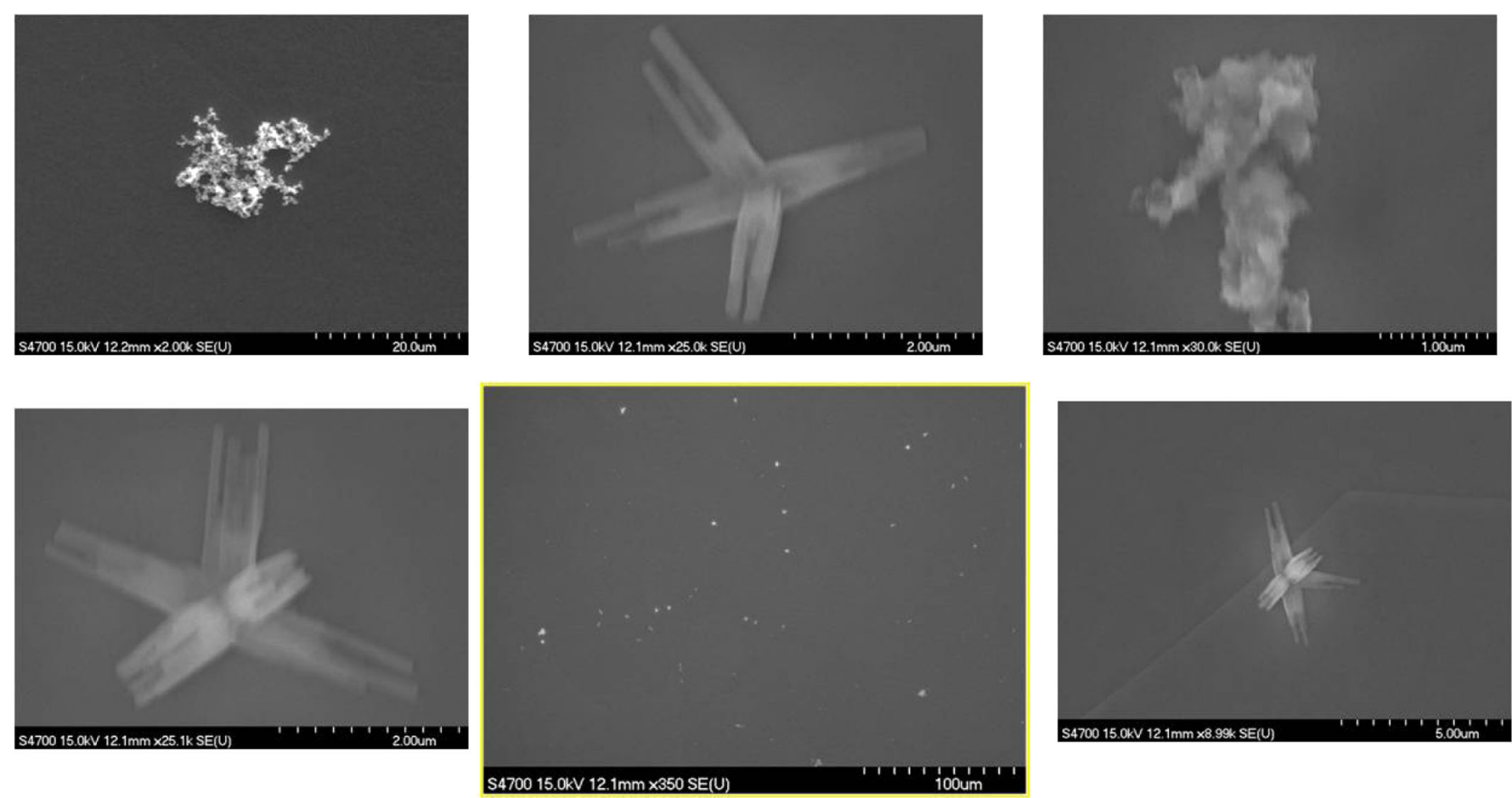

FIG. 12. Nb-O granules and crystallite-like particles were found on one area (the center figure in row 2) of sample 6.

presented by a red curve in the middle of Fig. 11. The sulfur peak at $2.3 \mathrm{keV}$ can be easily identified. The atomic percentage of sulfur is $5.32 \%$ after commercial EDAX software evaluation. Considering the EDX profiling depth is $1 \mu \mathrm{m}$, the sulfur distribution is relatively deep in the granule. For comparison, the third EDX window was set at a spot $50 \mu \mathrm{m}$ away from the granule. That spot is ultraflat and has no particles at all. The spectrum from the flat plane is presented by a black curve in the bottom of Fig. 11. This curve has no sulfur peak and its oxygen peak is very small. This spectrum is reasonable because the natural oxidized niobium surface only has a few of $\mathrm{nm}$ in thickness.

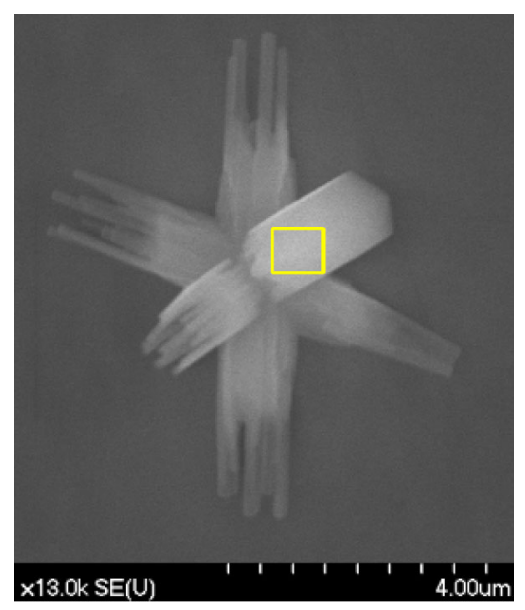

FIG. 13. SEM micrograph of one crystallite-like particle. The yellow box labels an area surveyed by EDX (see Fig. 14).
Figure 11 gives an overview of elemental concentration on various spots of the granule and on a normal EP'ed surface.

On one area of sample 6 , besides the omnipresent $\mathrm{Nb}$ granules, unusual crystallite-like particles (see Figs. 12 and 13) were observed. An EDX survey window (in yellow) is set at the crystallite-like particle (Fig. 13). Figure 14 is the EDX spectrum that revealed elements of $\mathrm{N}, \mathrm{O}, \mathrm{S}, \mathrm{Fe}$, and

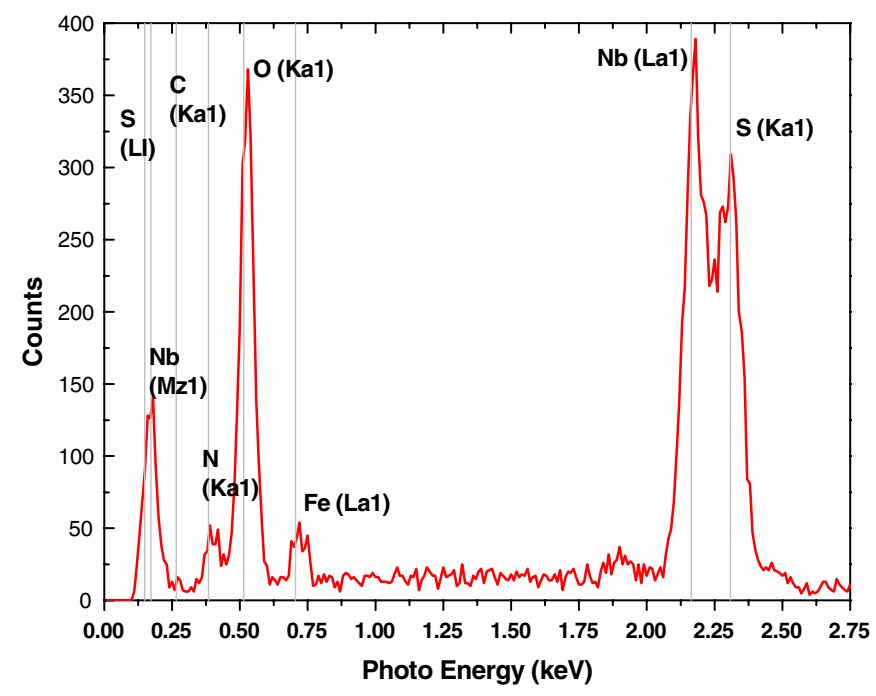

FIG. 14. EDX spectrum surveyed from the yellow box (Fig. 13). It contains $\mathrm{S}, \mathrm{Fe}$, and $\mathrm{N}$ elements besides $\mathrm{Nb}$ and $\mathrm{O}$. The origin of $\mathrm{Fe}$ and $\mathrm{N}$ are unknown. The elemental ratio of $\mathrm{N}, \mathrm{O}, \mathrm{S}, \mathrm{Fe}$, and $\mathrm{Nb}$ is 19:72:5:3:1 after commercial EDAX software evaluation. 
TABLE II. Summary of the three samples investigated by AES and SEM/EDX tools.

\begin{tabular}{llll}
\hline \hline Sample & EP'ed location in SRF cavity & AES investigation summary & SEM/EDX investigation summary \\
\hline$\# 2$ & Equator & 5 spots surveyed. No S was found & Nb-O granule was found \\
$\# 4$ & Iris & 5 spots survey. S was found at 4 spots & Nb-O granule was found \\
$\# 6$ & Beam pipe & 11 spots survey. S was found at 3 spots & $\begin{array}{l}\text { Nb-O granule was found with S; crystallite-like } \\
\text { particles with Fe, S, N, Nb, and O elements } \\
\end{array}$ \\
& & were observed \\
\hline \hline
\end{tabular}

$\mathrm{Nb}$ on that area. The elemental ratio of $\mathrm{N}, \mathrm{O}, \mathrm{S}, \mathrm{Fe}$, and $\mathrm{Nb}$ is 19:72:5:3:1 after commercial EDX software evaluation. Similar crystallite features have not been observed on the other EP'ed samples. It is an atypical phenomenon after EP. The source and formation mechanism of the "microflower-like" crystallite particles are unknown. Had sharp edged particles left on a real SRF cavity surface, they might degrade the cavity performance as field emission sources.

\section{SUMMARY}

Three niobium samples treated in a single cell SRF niobium cavity as a witness of electropolishing (EP) processing have been surveyed by AES and SEM/EDX. Oxygen-enriched niobium granules (like sintered powder) have been observed on all samples. Their crystal structure and formation mechanism are unknown. A few atomic layers of sulfur were observed covering the niobium samples surface nonuniformly. Some areas of the microgranules could harbour sulfur deeply $(>10 \mathrm{~nm}$, see Fig. 8). Crystallite particles containing unexpected impurities (e.g. Fe) were found on one sample. Table II summarized the surface characterization results in general. These results suggest that post-EP cleaning of real niobium cavities should aim for removal of both sulfur residues and oxygen-enriched niobium granules in order to reduce field emitters.

\section{ACKNOWLEDGMENTS}

We acknowledge Dr. Seo Kang of Norfolk State University for his support on AES experiments. We thank the College of William \& Mary for the support on SEM experiments. This manuscript has been sponsored by Jefferson Science Associates, LLC under U.S. DOE Contract No. DE-AC05-06OR23177.
[1] P. A. Jacquet, Nature (London) 135, 1076 (1935).

[2] H. Diepers, O. Schmidt, H. Martens, and F. S. Sun, Phys. Lett. A 37, 139 (1971).

[3] K. Saito, in Proceedings of the 20th Particle Accelerator Conference, Portland, OR, 2003 (IEEE, New York, 2003), p. 462 [http://accelconf.web.cern.ch/AccelConf/p03/ PAPERS/ROAA002.PDF].

[4] F. Eozénou, A. Aspart, C. Antoine, and B. Maliki, CARE Report No. 06-10-SRF, 2006.

[5] F. Eozénou, C. Antoine, A. Aspart, S. Berry, J. F. Denis, and B. Malki, in Proceedings of the 12th SRF Workshop, Ithaca, NY (Cornell University, Ithaca, NY, 2005), ThP02 [http://www.lns.cornell.edu/public/SRF2005/pdfs/ ThP02.pdf].

[6] A. Aspart, F. Eozenou, and C. Antoine, in Proceedings of the 12th SRF Workshop, Ithaca, NY (Ref. [5]), ThP03.

[7] R.L. Geng, in Proceedings of the 14th International Conference on RF Superconductivity, Berlin Germany, 2009, TUPPO015 [Helmholtz Centre Berlin (HZBBerichte), Berlin, Germany, 2009] [http://accelconf.web .cern.ch/AccelConf/srf2009/index.htm].

[8] Charles Reece and Hui Tian, in Proceedings of the XXV Linear Accelerator Conference (LINAC 10), Tsukuba, Japan [High Energy Accelerator Research Organization (KEK), Japan, 2010], THP010 [http://spms.kek.jp/pls/ linac2010/TOC.htm].

[9] R. J. Noer, Ph. Niedermann, N. Sankarraman, and Q. Fischer, J. Appl. Phys. 59, 3851 (1986).

[10] Ph. Niedermann, N. Sankarraman, R. J. Noer, and O. Fischer, J. Appl. Phys. 59, 892 (1986).

[11] Lawrence E. Davis, Handbook of Auger Electron Spectroscopy: A Reference Book of Standard Data for Identification and Interpretation of Auger Electron Spectroscopy Data (Physical Electronics, New York, 1996), ISBN 13: 978-0964812406. 